\title{
A highly miniaturized electron and ion energy spectrometer prototype for the rapid analysis of space plasmas
}

\author{
R. Bedington ${ }^{2, a, b)}$, D.O. Kataria ${ }^{1)}$ and A. Smith ${ }^{1)}$ \\ ${ }^{1}$ Mullard Space Science Laboratory, University College London, Holmbury St Mary, \\ RH5 6NT, United Kingdom \\ ${ }^{2}$ Institute of Space and Aeronautical Science, Japan Aerospace Exploration Agency, \\ Sagamihara, 229-8510, Japan
}

MEMS (Micro Electro-Mechanical Systems) plasma analyzers are a promising possibility for future space missions but conventional instrument designs are not necessarily well suited to micro-fabrication.

Here, a candidate design for a MEMS-based instrument has been prototyped using EDM (electron-discharge machining). The device features ten electrostatic analyzers that, with a single voltage applied to it, allows five different energies of electron and five different energies of positive ion to be simultaneously sampled. It has been simulated using SIMION and the electron response characteristics tested in an instrument calibration chamber. Small deviations found in the electrode spacing of the as-built prototype were found to have some effect on the electron response characteristics but do not significantly impede its performance.

\footnotetext{
${ }^{a}$ Author to whom correspondence should be addressed. Electronic mail: r.bedington@stp.isas.jaxa.jp

${ }^{b}$ This research was performed while R. Bedington was at Mullard Space Science Laboratory.
} 


\section{INTRODUCTION}

Plasmas in space, and particularly those around magnetized planets, exhibit complex behaviors that are usually measured only with single, or small numbers of spacecraft using complex and often bulky instrumentation. Since space plasmas vary both temporally and spatially, multipoint measurements of this environment are essential for a comprehensive understanding its processes. ${ }^{1}$

To make numerous concurrent multipoint measurements of this environment, large numbers of sensors are required, but naturally the launch costs incurred scale with the payload mass. While some missions of this nature have been proposed to various space agencies, ${ }^{1-4}$ they have not been selected. Significantly miniaturized instrumentation would allow for cost reductions that may make these missions more attractive. Such devices would also find applications in CubeSats and other nanosats for which multipoint missions of a similar nature are already being developed. ${ }^{5}$

Plasma observing suites usually include energy spectrometers for electrons and ions with a few $\mathrm{eV}$ to a few tens keV kinetic energies. These devices typically consist of electrostatic deflection electrodes (commonly in a cylindrical ${ }^{6}$, spherical ${ }^{7}$ or top-hat configuration ${ }^{8}$ ) coupled with a detector (most usually microchannel plate-based ${ }^{9}$ ).

Many miniaturized versions of these sensors have been produced by various groups, sometimes by using novel new electrode arrangements ${ }^{10}$, often by scaling down conventional designs ${ }^{11}$ and also by combining functions (e.g. electron and ion measuring) into smaller packages ${ }^{12}$. While a large variety of electrode designs exist, they are usually machined from aluminum using conventional machining techniques. The amount by which these electrodes can be miniaturized is limited by the manufacturing tolerances of these techniques as well as by the dimensions of the mounting screws.

A solution for further miniaturizing electrodes is the use of MEMS (Micro Electro Mechanical Systems). MEMS techniques allow for the production of very small scale structures with very high accuracy, albeit limited to fairly simple shapes. MEMS components have found various applications in space ${ }^{13,14}$, including within low-energy plasma spectrometers ${ }^{15-18}$. Instruments of this kind have used novel, but unfocussed, electrode geometries, replicated multiple times to achieve acceptable sensitivities. MEMS have also been used for ground based electrostatic analyzer instruments, where simple focusing geometries have been demonstrated ${ }^{19}$.

For space science applications focusing geometries with uniform responses and high sensitivities/geometric factors ${ }^{20}$ are often desirable and for MEMS fabrication novel electrode geometries and arrangements will be required.

The CATS (cylindrical and tiny spectrometer) prototype is a development towards such a MEMS plasma spectrometer. While it is not MEMS itself, its highly miniaturized design is well suited to micro-fabrication and would allow for low resource, high time resolution energy analysis of electrons and ions simultaneously. 


\section{CATS - CYLINDRICAL AND TINY SPECTROMETER}

Cylindrical electrostatic analyzers consist of two curved parallel electrodes at different electric potentials. Ions or electrons enter the analyser at one end and either follow a curved path through to the other end or collide with the walls of the analyser, depending on their initial kinetic energy to charge ratio and the voltages on the electrodes. ${ }^{21}$

CATS contains ten concentric 90-degree cylindrical electrostatic analyzers, ten channels, that measure five different energies of electrons and five different energy/charge ratios of [positive] ions simultaneously, thus potentially allowing for the rapid acquisition of plasma energy spectra. A schematic of the electrode arrangement is shown in figure 1.

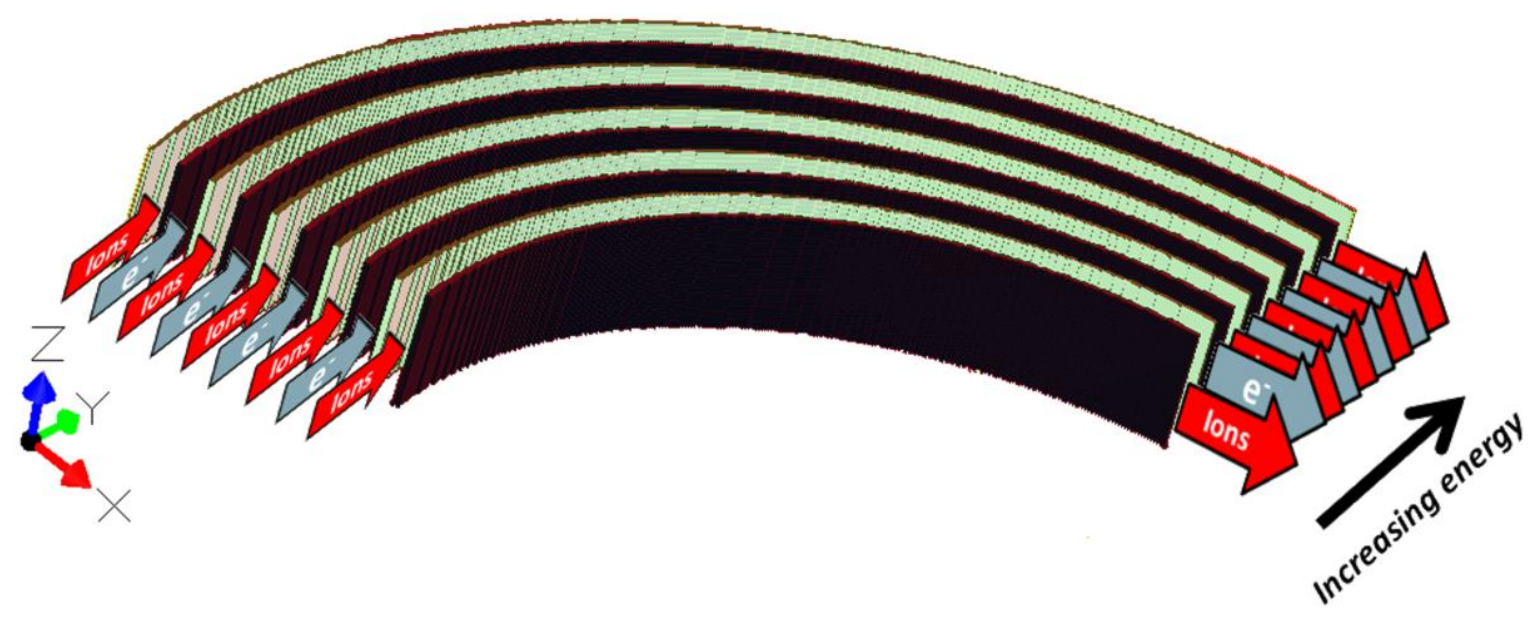

Figure 1 - Schematic of CATS electrode arrangement [innermost electron channel is not shown]. The electrodes are colored dark and light to represent the two different electric potentials used. The black electrodes are more negative so the ions and electrons are selected accordingly. For incoming particles the central beam direction is defined as parallel to the $\mathrm{Y}$ axis, with elevation angle as the angle between $\mathrm{Y}$ and $\mathrm{X}$, and azimuth angle as the angle between $\mathrm{Y}$ and $\mathrm{Z}$.

Since the outer (larger) radius electrode of one channel is also the inner (smaller) radius electrode of the adjacent channel, the channels are alternately electron and ion analyzers.

The peak energy/charge of each channel can be approximated by equating the electric and centripetal forces of a charged particle traveling on a circular orbit in the center of that channel. The following equation shows this in relation to $K$ (the analyzer constant), the constant ratio of the peak energy/charge value to the potential difference between the electrode plates.

$$
K=\frac{E_{\text {selected }} / Q}{V_{\text {applied }}}=\frac{R_{0}}{2 \times \Delta R}
$$

When $R_{0} \gg>R$.

Where $E_{\text {selected }} / Q$ is the particle energy/charge ratio selected by the analyzer, $V_{\text {applied }}$ is the potential difference between the analyzer plates, $R_{0}$ is the radius of the central trajectory and $\Delta R$ is the gap between the plates. Since in CATS $V_{\text {applied }}$ and $\Delta R$ are the same for all channels, 
the peak energy of each channel increases linearly channel to channel with increasing radius (i.e. in figure 1, increasingly energetic particles are found from left to right at the exit).

The basic construction of the cylindrical and tiny spectrometer is shown in figure 2 .

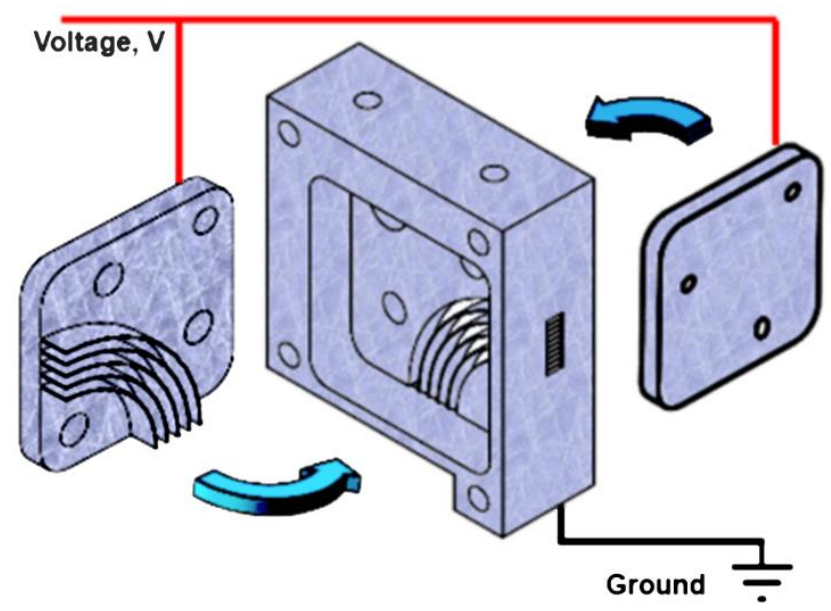

Figure 2 - the electrode components of CATS; the analyzer voltage is applied to the left and right pieces, the central component is grounded. For clarity the fixing screws, insulating dowels and covers are not shown.

This shows the three components that form the electrodes; the finned section on the left locates into the slots in the grounded electrode in the center, bisecting each slot to create ten concentric analyzer channels. The plate on the right closes the box so that the only access to the channels is through the aperture holes on the front and bottom of the central grounded electrode.

The electrodes are held in place and in alignment by screws within insulating dowels (not shown). To prevent high voltages being exposed outside the analyzer, the outer electrodes are covered by larger grounded plates (also not shown) that mount onto the central grounded electrode. In this prototype device the fins and slots were created in aluminum using electrondischarge-machining (EDM) - see figure 3 - as this was found to be more cost-effective than MEMS for a proof-of-concept model. A MEMS version of the device might be made with LIGA or with deep reactive ion etching (DRIE) in silicon.

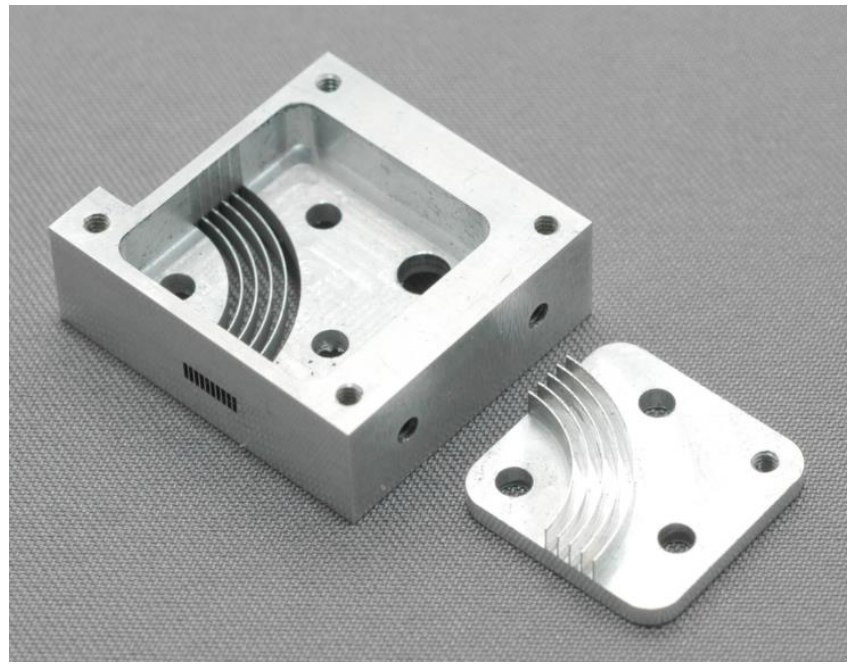

Figure 3 - EDM fabricated components of CATS, approx. $2 \mathrm{~cm} \times 2 \mathrm{~cm} \times 0.75 \mathrm{~cm}$. 


\section{CATS PERFORMANCE}

The CATS analyzer was modelled using SIMION ion optics software ${ }^{22}$ and the prototype was tested with a mono-energetic, flood electron beam and a nickel, beta source in the MSSL electron instrument calibration vacuum chamber (the facility is described in other publications ${ }^{23,24}$ ). Initially a Dr Sjuts KBL408 ceramic CEM (channel electron multiplier) was used to detect electrons exiting the analyser. It was mounted in a housing with a collimating entrance aperture - figure 4 - that could be positioned in front of one of the CATS apertures by use of a translation stage.

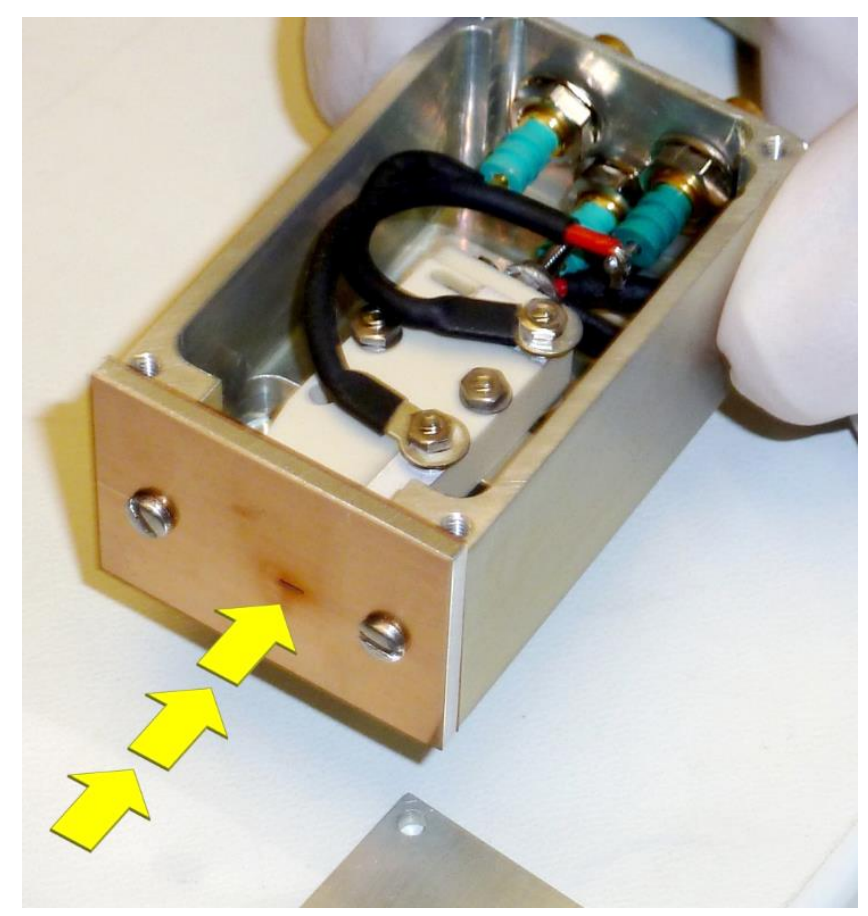

Figure 4 - A CEM that could be positioned in front of individual CATS exit apertures. Yellow arrows indicate where electrons would access the CEM through a collimated aperture.

Latterly an e2v CCD64; a back-thinned, ion-implanted CCD was also used to image the electron footprint from all channels as well as to test the response of the CCD to low energy electrons. This has previously been described in Bedington et al. ${ }^{25}$

While the actual instrument parameters vary slightly from channel to channel, and between laboratory and simulation, the summarized results are shown in table 1. 
Table I. Instrument parameters for as-designed CATS.

\begin{tabular}{|l|l|}
\hline \hline K factors & $10.5-16.5$ \\
\hline Nominal $\Delta R$ & $0.3 \mathrm{~mm}$ \\
\hline Nominal $R_{0}$ (of smallest channel) & $6.45 \mathrm{~mm}$ \\
\hline Nominal channel wall thickness & $0.1 \mathrm{~mm}$ \\
\hline Energy resolution $\left(E_{F W H M} / E_{P e a k}\right)$ & $6-8 \%$ \\
\hline Geometric factor (per channel) & $10^{-6} \mathrm{~cm}^{2} \mathrm{sr} \mathrm{eV} / \mathrm{eV}$ \\
\hline Geometric factor (total) & $10^{-5} \mathrm{~cm}^{2} \mathrm{sr} \mathrm{eV} / \mathrm{eV}$ \\
\hline Angular resolution & $3^{\circ} \times 6-8^{\circ}$ \\
\hline Mass of analyser head & $50 \mathrm{~g}$ \\
\hline \hline
\end{tabular}

Under visual inspection, the width of the channels $(\Delta R)$ was found to vary along their length, an effect inadvertently introduced by the EDM manufacturing process. This variation causes some discrepancies between laboratory and simulation data - the resultant k-factor was found to correspond to the smallest $\Delta R$ in any given channel, i.e. the narrowest point. Figure 5 compares the expected response at different $V_{\text {applied }}$ settings for SIMION data and laboratory data. In this figure the laboratory $\mathrm{k}$ factors have been multiplied by 1.06 as a first order correction to the channel thickness variations.

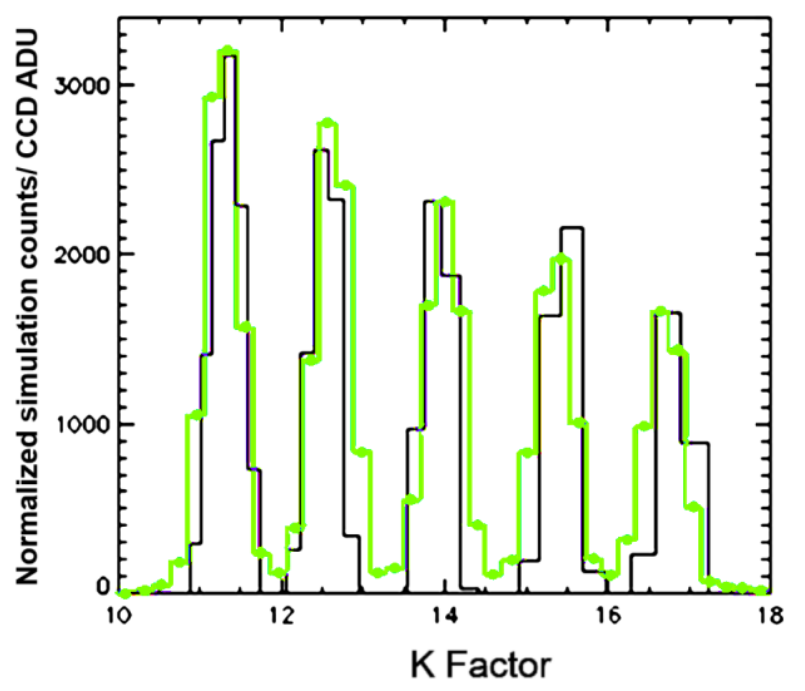

Figure 5 - SIMION simulation (green with dots) and long acquisition mode ${ }^{24}$ CATS+CCD data (black) for five of the CATS analyzer channels under a $2.5 \mathrm{keV}$ electron beam. Absolute laboratory measurements were not available so the vertical scale has been normalized to the central channel to allow the relative heights to be compared with the simulation data. A horizontal scaling factor of 1.06 has been applied to correct for the electrode spacing variations in the as-built geometry.

The five peaks in figure 5 correspond to the monoenergetic electrons passing through five of the CATS channels when varying negative voltages were applied to the finned electrode. Higher peaks correspond to higher geometric factors and these are seen at the lower $\mathrm{k}$ factors, i.e. the smaller radiuses. This occurs due to the changing $\Delta R$ to $R_{0}$ ratio between channels.

Figure 6 shows results for a fixed $V_{\text {applied }}$ and varying beam input angles, from which the shapes of the angular responses can be seen to be largely as expected. 

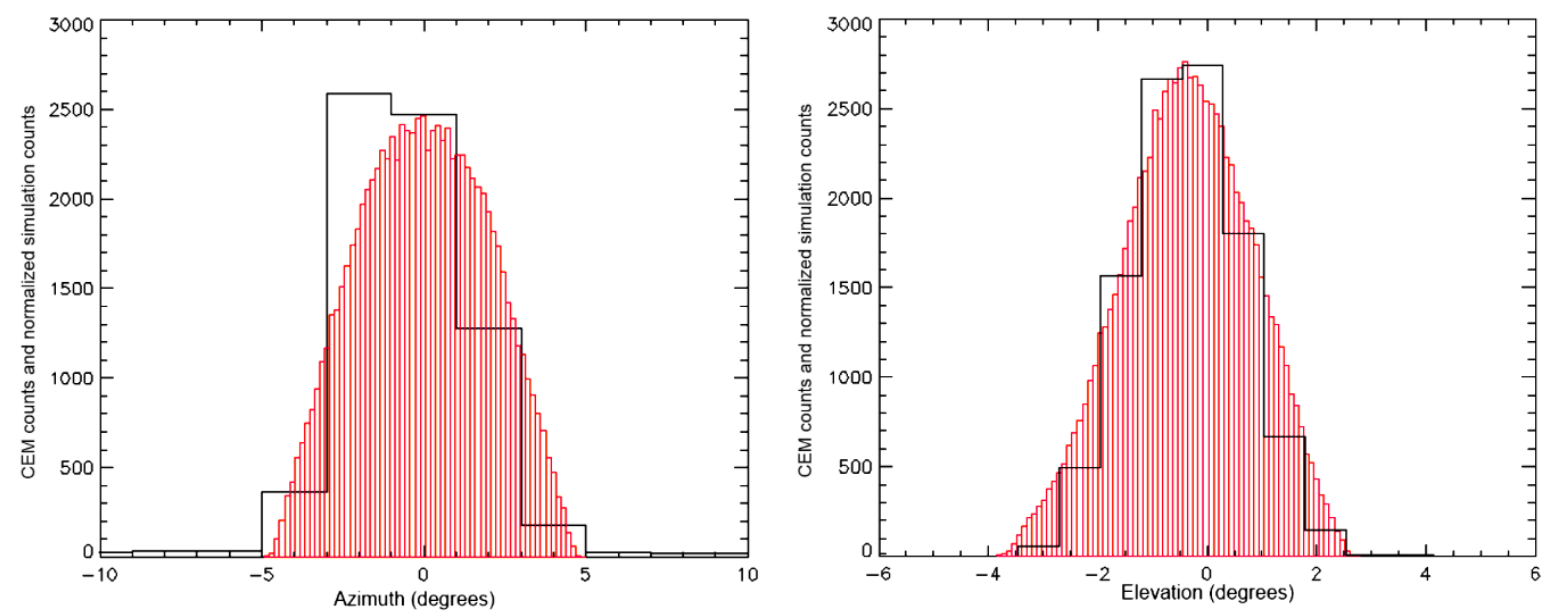

Figure 6 - Angular responses for a single CATS analyzer channel ( $\mathrm{k}$ factor $=15$ ) with a $300 \mathrm{eV}$ electron beam and CEM detector (black line) and corresponding simulation data (red columns). The peak heights have been approximately normalized and the $\mathrm{x}$ axes have been shifted to account for offsets.

The absolute azimuth positions were subject to a systematic offset of $-1^{\circ}$ across all channelsa 1 degree rotation from $\mathrm{Z}$ to $\mathrm{Y}$ about $\mathrm{X}$ in figure 1 . This would appear to be caused by a slight offset in the mounting arrangement or calibration, since the experiment was magnetically shielded in a mu-metal case, and since a different systematic azimuthal offset was encountered in the CCD-based test (which had a different mounting setup).

While the elevation position had a comparable offset, the channel width variations in the asbuilt model added an additional component of up to 2 degrees to the actual elevation peak shift. Accordingly in figure 6 the CEM data peak has been aligned to the simulation data peak. Since CATS is a 90 degree cylindrical analyzer, this peak elevation is very slightly negative.

\section{DISCUSSION}

The CATS project was focused on technology development and so the instrument parameters were not specifically targeted towards a particular plasma environment or space mission; cost and manufacturing considerations drove the design. A scientific instrument based on the CATS design would require optimized instrument parameters appropriate to the plasma environment that it is to study.

For example, with the current design the CATS geometric factors are relatively small and would be best suited to plasmas with relatively high fluxes. The geometric factors could be increased however by reducing the analyzer bending angle (at the cost of a worsening of the energy resolution) or by widening the channels in width $(\Delta R)$ or depth (affecting the angular resolutions accordingly). It should be noted though that since miniaturized analyzers have miniaturized apertures, their geometric factors are intrinsically lower than larger scale instruments ${ }^{26}$. This limitation can be mitigated in part through the use of multiple sensor heads or larger arrays of channels. For reasons of surface area to volume scaling differences, large numbers of miniaturized analyzers still offer appreciable spacecraft resource savings when compared to a larger scale instrument of equivalent aperture area ${ }^{15}$. 
Various arrangements of multiple CATS heads have been conceived to provide for wider fields of view and greater total geometric factors, as well as MEMS implantations of the design that offer the potential for more compact designs made to greater accuracy.

Although CATS was not intended as a flight component, the electron beam tests had proven its functionality and provided an approximate calibration. Accordingly it was used in PoleCATS (Polar test of CATS): a student sounding rocket payload that used CATS and the CCD (with Peltier cooling). This flew from Esrange, Sweden on the REXUS 14 mission (rocket experiments for university students) in May 2013. The PoleCATS experiment is described in detail in Lee et al. ${ }^{27}$.

A clear requirement for a future iteration of CATS would be for an optical blacking coating to be applied within the channel to reduce stray light reflections reaching the detector and thus adding noise to the data. While an Ebanol-C process is often used to achieve this ${ }^{28}$, the fine filaments this creates were deemed to cause a risk of electrical arcing within the narrow CATS channels and a lower profile coating should be sought instead.

\section{ACKNOWLEDGEMENTS}

CATS Technology development funded by STFC rolling grant.

R.B CATS PhD studentship ${ }^{29}$ funded by STFC CASE with Astrium UK, now at ISAS JAXA and partly supported by JSPS Grant-in-Aid for JSPS Fellows Grant Number 24.02792

With thanks to Yoshifumi Saito for his advice.

\section{REFERENCES}

${ }^{1}$ V. Angelopoulos and H.E. Spence. Sun-Earth Plasma connections, (American Geophysical Union, Washington DC, 1999) p247.

${ }^{2}$ S. J. Schwartz, T. Horbury, C. Owen, W. Baumjohann, R. Nakamura, P. Canu, A. Roux, F. Sahraoui, P. Louarn, J.-A. Sauvaud, J.-L. Pinçon, A. Vaivads, M. F. Marcucci, A. Anastasiadis, M. Fujimoto, P. Escoubet, M. Taylor, S. Eckersley, E. Allouis, and M.-C. Perkinson. Experimental Astronomy, 23, 1001 (2008).

${ }^{3}$ M. Fujimoto, Y. Tsuda, Y. Saito, I. Shinohara, T. Takashima, A. Matsuoka, H. Kojima, and Y. Kasaba, AIP Conf. Proc. 29, 1144 (2009).

${ }^{4}$ J. Fennell, H. Spence, T. Moore, and J. Galloway. Cluster-II Workshop Multiscale/Multipoint Plasma Measurements, London, UK 1999, ESA Publications SP-449, 235 (2000).

${ }^{5}$ E. Gill, P. Sundaramoorthy, J. Bouwmeester, B. Zandbergen, R. Reinhard, Acta Astronautica, 82, 110 (2013). 
${ }^{6}$ M. Neugebauer and C.W. Snyder, JGR, 71, 19 (1966).

${ }^{7}$ S. J. Bame, D. J. McComas, M. F. Thomsen, B. L. Barraclough, R. C. Elphic, J. P. Glore, J. T. Gosling, J. C. Chavez, E. P. Evans, F. J. Wymer, Rev. Sci. Instrum, 64, 1026 (1993)

${ }^{8}$ C. W. Carlson, D.W. Curtis, G. Paschmann, and W. Michael, Adv. Space Res. 2, 67 (1983).

${ }^{9}$ J. Ladislas Wiza. Nuclear Instruments and Methods, 162, 587 (1979).

${ }^{10}$ P.L. Koehn, T.H. Zurbuchen, G. Gloeckler, R.A. Lundgren, and L.A. Fisk, Meteoritics \& Planetary Science, 37, 1173, (2002).

${ }^{11}$ D. T. Young, (R. F. Pfaff, J. E. Borovsky, and D. T. Young, editors), Measurement Techniques in Space Plasmas - Particles (American Geophysical Union, Washington DC, 1998), p313.

${ }^{12}$ J. L. Burch, R. Goldstein, T. E. Cravens, W. C. Gibson, R. N. Lundin, C. J. Pollock, J. D. Winningham, and D. T. Young. Space Science Reviews, 128, 697 (2006).

${ }^{13}$ S. Janson, H. Helvajian, S. Amimoto, G. Smit, D. Mayer, and S. Feuerstein. In Aerospace Conference, 1998. Proceedings.,IEEE, 1, 409 (1998).

${ }^{14} \mathrm{R}$. Osiander, M. Darrin, and J. Champion. MEMS and Microstructures in aerospace applications. (Taylor \& Francis, Boca Raton, FL) 2006.

${ }^{15}$ R. Stalder, S. Boumsellek, T. Van Zandt, T. Kenny, M. Hecht, and F. Grunthaner. Journal of Vacuum Science \& Technology A: Vacuum, Surfaces, and Films, 12, 2554 (1994).

${ }^{16}$ C. L. Enloe, L. H. Krause, R. K. Haaland, T. T. Patterson, C. E. Richardson, C. C. Lazidis, and R. G. Whiting. Rev. Sci. Instrum,

74, 1192 (2003).

${ }^{17}$ D. M. Wesolek, F. A. Hererro, R. Osiander, and M. A. G. Darrin, Journal of Microlithography, Microfabrication, and Microsystems, 4, 041403 (2005).

${ }^{18}$ H. S. Feldmesser, M. A. G. Darrin, R. Osiander, L. J. Paxton, a. Q. Rogers, J. A. Marks, M. G. McHarg, R. L. Balthazor, L. H. Krause, and J. G. FitzGerald. Proceedings of SPIE, 7691, 76910 (2010).

${ }^{19}$ E. Wapelhorst, J. Hauschild, and J. Muller, Sensors and Actuators A: Physical, 138, 22 (2007).

${ }^{20}$ G. A. Collinson, J. C. Dorelli, L. A. Avanov, G. R. Lewis, T. E. Moore, C. Pollock, D. O. Kataria, R. Bedington, C. S. Arridge, D. J. Chornay, U. Gliese, A. Mariano, A. C. Barrie, C. Tucker, C. J. Owen, A. P. Walsh, M. D. Shappirio, and M. L. Adrian, Rev. Sci. Instrum. 83, 033303 (2012).

${ }^{21}$ Mikhail Yavor, Advances in Imaging and Electron Physics Volume 157, (Elsevier, 2009), p.213. 
${ }^{22}$ R. Bedington, D. O. Kataria, A. Smith, and S. Eckersley. Proceedings of the Symposium on Small Satellite Systems and Services (4S), Funchal, Portugal 2010, ESA Publications (2010).

${ }^{23}$ A. Johnstone, C. Alsop, S. Burge, P. Carter, A. Coates, A. Coker, A. Fazakerley, M. Grande, R. Gowen, C. Gurgiolo. Space Science Reviews, 79, 351 (1997).

${ }^{24}$ G. Lewis, C. Arridge, D. Linder, L. Gilbert, D. Kataria, A. Coates, A. Persoon, G. Collinson, N. André, P. Schippers, J. Wahlund, M. Morooka, G. Jones, A. Rymer, D. Young, D. Mitchell, A. Lagg, and S. Livi. Planetary and Space Science, 58, 427 (2010).

${ }^{25}$ R. Bedington, D. Kataria, and D.Walton. Journal of Instrumentation, 7, C01079 (2012).

${ }^{26}$ H.O. Funsten and D.J. McComas, (R. F. Pfaff, J. E. Borovsky, and D. T. Young, editors), Measurement Techniques in Space Plasmas - Particles (American Geophysical Union, Washington DC, 1998), page 157.

${ }^{27}$ A. Lee, R. Bedington, L. Comandar, A. Daurskikh, M. Hills, D. Hu, R. Lee, and T. Nordheim, The proceedings of the 21st ESA PAC Symposium, Thun Switzerland, 2013, edited by L. Ouwehand (ESA Communications SP-721, 2013), pp. 469-472.

${ }^{28}$ C. Alsop, S. Scott, and L. Free. (R. F. Pfaff, J. E. Borovsky, and D. T. Young, editors), Measurement Techniques in Space Plasmas - Particles (American Geophysical Union, Washington DC, 1998), page 269.

${ }^{29}$ R. Bedington, Ph.D. thesis, University College London, 2012. 